\title{
Preliminary study of the $\bar{K} N$ interaction in a chiral constituent quark model
}

\author{
F. Huang ${ }^{1}$, W.L. Wang ${ }^{2}$, Z.Y. Zhang ${ }^{3}$, and Y.W. $\mathrm{Yu}^{3}$ \\ ${ }^{1}$ CCAST (World Laboratory), P.O. Box 8730, Beijing 100080, China \\ ${ }^{2}$ Institute of Particle Physics, Huazhong Normal University, Wuhan 430079, China \\ ${ }^{3}$ Institute of High Energy Physics, P.O. Box 918-4, Beijing 100049, China
}

\begin{abstract}
A preliminary investigation of $\bar{K} N$ interaction is performed within a chiral constituent quark model by solving the resonating group method (RGM) equation. The model parameters are taken from our previous work, which gave a satisfactory description of the $S_{-}, P_{-}, D_{-}, F$-wave $K N$ scattering phase shifts. The channel-coupling between $\bar{K} N, \pi \Lambda$ and $\pi \Sigma$ is considered, and the scattering phase shifts as well as the bound-state problem of $\bar{K} N$ are dynamically studied. The results show that the $S$-wave $\bar{K} N$ interaction in the isospin $I=0$ channel is attractive, and in the extended chiral $\mathrm{SU}(3)$ quark model such an attraction can make for a $\bar{K} N$ bound state, which appears as a $\pi \Sigma$ resonance in the coupled-channel calculation, while the chiral SU(3) quark model cannot accommodate the existence of a $\bar{K} N$ bound state. It seems that the vector meson exchanges are necessary to be introduced in the quark-quark interactions if one tries to explain the $\Lambda(1405)$ as a $\bar{K} N$ bound state or a $\pi \Sigma-\bar{K} N$ resonance state.

PACS numbers: 13.75.Jz, 12.39.-x, 21.45.+v
\end{abstract}

Nowadays, due to the complexity of the nonperturbative QCD effect, people still need QCD-inspired models to be a bridge connecting the QCD fundamental theory and the experimental observables. Among these phenomenological models, the chiral SU(3) quark model and the extended chiral SU(3) quark model have been quite successful in reproducing the energies of the baryon ground states, the binding energy of the deuteron, the nucleon-nucleon $(N N)$ and kaon-nucleon $(K N)$ scattering phase shifts, and the hyperon-nucleon $(Y N)$ cross sections [1, 2, 3, 4]. In the original chiral $\mathrm{SU}(3)$ quark model, the quark-quark interaction contains confinement, one gluon exchange (OGE) and boson exchanges stemming from scalar and pseudoscalar nonets, and meanwhile, it is found that the short range quark-quark interaction is provided by OGE and quark exchange effects. In the extended chiral SU(3) quark model, the vector meson exchanges are included and consequently the OGE is largely reduced, thus the short range quark-quark interaction is now dominantly provided by vector-meson exchange and quark exchange effects.

As a matter of fact, it is still a controversial problem for low-energy hadron physics whether gluon or Goldstone boson is the proper effective degree of freedom besides the constituent quark. Glozman and Riska proposed that the Goldstone boson is the only other proper effective degree of freedom [5]. But Isgur insisted that the OGE governs the baryon structure [6]. Anyway, it is still a challenging problem in the low-energy hadron physics whether OGE or vector-meson exchange is the right mechanism or both of them are important for describing the short-range quark-quark interaction.

In the past few years, by use of the resonating group method (RGM), we have dynamically studied the $N N$ and $K N$ scattering phase shifts, and the $\Omega \Omega, N \bar{\Omega}, \Delta K$, $\Lambda K$ and $\Sigma K$ interactions within both the chiral $\mathrm{SU}(3)$ quark model and the extended chiral SU(3) quark model $[1,2,3,4,7,8,[9]$. It was found that though the mech- anisms of the short range quark-quark interaction are quite different, these two models give quite similar results for all those hadron-hadron systems. To get some more definite information about the short range quarkquark interaction mechanisms, it seems interesting and necessary to investigate some special systems where the chiral SU(3) quark model and the extended chiral SU(3) quark model give different contributions.

One notices that the $K N$ and $\bar{K} N$ are interesting cases, since there is a close connection of the vectormeson exchanges between the $K N$ and $\bar{K} N$ interactions due to $G$-parity transition on hadron level. Specially, the $\omega$ exchange is repulsive for $K N$ while attractive for $\bar{K} N$, because of the negative $G$ parity of the $\omega$ meson. Our previous work [4] has already shown that for the $K N$ system the scattering phase shifts from the chiral $\mathrm{SU}(3)$ quark model and the extended chiral SU(3) quark model are quite similar, which means that in the $K N$ system the repulsion of OGE can be substituted by that of vector meson exchange. Thus it is naturally expected that for the $\bar{K} N$ system the results from the chiral $\mathrm{SU}(3)$ quark model and the extended chiral SU(3) quark model must be quite different since in the $\bar{K} N$ system the OGE vanishes while the vector-meson exchange is attractive.

In this work, we perform a preliminary study of the $\bar{K} N$ interaction in both the chiral SU(3) quark model and the extended chiral SU(3) quark model. Let's first briefly review the models (the detailed formula of which can be found in Ref. [4]). The total Hamiltonian of baryon-meson systems can be written as

$$
H=\sum_{i=1}^{5} T_{i}-T_{G}+\sum_{i<j=1}^{4} V_{i j}+\sum_{i=1}^{4} V_{i \overline{5}},
$$

where $T_{G}$ is the kinetic energy operator for the center-ofmass motion, and $V_{i j}$ and $V_{i \overline{5}}$ represent the quark-quark and quark-antiquark interactions, respectively,

$$
V_{i j}=V_{i j}^{\mathrm{OGE}}+V_{i j}^{\mathrm{conf}}+V_{i j}^{\mathrm{ch}},
$$


where $V_{i j}^{\mathrm{OGE}}$ is the OGE interaction, $V_{i j}^{\text {conf }}$ is the confinement potential, and $V_{i j}^{\text {ch }}$ is the chiral fields induced effective quark-quark potential. In the chiral SU(3) quark model, $V_{i j}^{\text {ch }}$ includes the scalar boson exchanges and the pseudoscalar boson exchanges,

$$
V_{i j}^{\text {ch }}=\sum_{a=0}^{8} V_{\sigma_{a}}\left(\boldsymbol{r}_{i j}\right)+\sum_{a=0}^{8} V_{\pi_{a}}\left(\boldsymbol{r}_{i j}\right),
$$

and in the extended chiral SU(3) quark model, the vector boson exchanges are also included,

$$
V_{i j}^{\text {ch }}=\sum_{a=0}^{8} V_{\sigma_{a}}\left(\boldsymbol{r}_{i j}\right)+\sum_{a=0}^{8} V_{\pi_{a}}\left(\boldsymbol{r}_{i j}\right)+\sum_{a=0}^{8} V_{\rho_{a}}\left(\boldsymbol{r}_{i j}\right) .
$$

Here $\sigma_{0}, \ldots, \sigma_{8}$ are the scalar nonet fields, $\pi_{0}, . ., \pi_{8}$ the pseudoscalar nonet fields, and $\rho_{0}, . ., \rho_{8}$ the vector nonet fields. $V_{i \overline{5}}$ in Eq. (1) represents the quark-antiquark interaction,

$$
V_{i \overline{5}}=V_{i \overline{5}}^{\mathrm{conf}}+V_{i \overline{5}}^{\mathrm{OGE}}+V_{i \overline{5}}^{\mathrm{ch}},
$$

with

$$
V_{i \overline{5}}^{\mathrm{ch}}=\sum_{j}(-1)^{G_{j}} V_{i 5}^{\mathrm{ch}, j}
$$

Here $(-1)^{G_{j}}$ represents the $G$ parity of the $j$ th meson. The expressions of the potentials can be found in the literature [3, 4, 7].

All the model parameters are taken to be the same as in Ref. [4], which gave a satisfactory description of the $S$-, $P-, D-, F$-wave $K N$ phase shifts. Here we briefly give the procedure for the parameter determination. The three initial input parameters are taken to be the usual values: $b_{u}=0.5 \mathrm{fm}$ for the chiral $\mathrm{SU}(3)$ quark model and $0.45 \mathrm{fm}$ for the extended chiral SU(3) quark model, $m_{u(d)}=313 \mathrm{MeV}$, and $m_{s}=470 \mathrm{MeV}$. The coupling constant for scalar and pseudoscalar chiral field coupling, $g_{\mathrm{ch}}$, is fixed by the relation

$$
\frac{g_{\mathrm{ch}}^{2}}{4 \pi}=\left(\frac{3}{5}\right)^{2} \frac{g_{N N \pi}^{2}}{4 \pi} \frac{m_{u}^{2}}{M_{N}^{2}}
$$

with the empirical value $g_{N N \pi}^{2} / 4 \pi=13.67$. The coupling constant for vector coupling of vector-meson field is taken to be $g_{\text {chv }}=2.351$, the same as used in the $N N$ case [2]. The OGE coupling constants and the strengths of confinement are fitted by baryon masses and their stability conditions. All the parameters are tabulated in Table I.

From Table I one can see that in the extended chiral $\mathrm{SU}(3)$ quark model, $g_{u}^{2}$ and $g_{s}^{2}$ are much smaller than the values in the chiral $\mathrm{SU}(3)$ quark model. This means that when the coupling of quarks and vector-meson field is considered, the coupling constants of OGE are greatly reduced by fitting the mass splits of $m_{\Delta}-m_{N}$ and $m_{\Sigma}-m_{\Lambda}$. Thus the OGE that plays an important role of the quarkquark short-range interaction in the original chiral SU(3)
TABLE I: Model parameters. The meson masses and the cutoff masses: $m_{\sigma^{\prime}}=980 \mathrm{MeV}, m_{\kappa}=1430 \mathrm{MeV}, m_{\sigma}=675$ $\mathrm{MeV}, m_{\epsilon}=980 \mathrm{MeV}, m_{\pi}=138 \mathrm{MeV}, m_{K}=495 \mathrm{MeV}$, $m_{\eta}=549 \mathrm{MeV}, m_{\eta^{\prime}}=957 \mathrm{MeV}, m_{\rho}=770 \mathrm{MeV}, m_{K^{*}}=892$ $\mathrm{MeV}, m_{\omega}=782 \mathrm{MeV}, m_{\phi}=1020 \mathrm{MeV}, \Lambda=1500 \mathrm{MeV}$ for $\kappa$ and $1100 \mathrm{MeV}$ for other mesons.

\begin{tabular}{lcc}
\hline \hline & $\chi$-SU(3) QM & Ex. $\chi$-SU(3) QM \\
\hline$b_{u}(\mathrm{fm})$ & 0.5 & 0.45 \\
$m_{u}(\mathrm{MeV})$ & 313 & 313 \\
$m_{s}(\mathrm{MeV})$ & 470 & 470 \\
$g_{u}^{2}$ & 0.7704 & 0.0748 \\
$g_{s}^{2}$ & 0.5525 & 0.0001 \\
$g_{\text {ch }}$ & 2.621 & 2.621 \\
$g_{\text {chv }}$ & & 2.351 \\
$a_{u u}^{c}\left(\mathrm{MeV} / \mathrm{fm}^{2}\right)$ & 55.7 & 60.3 \\
$a_{u s}^{c}\left(\mathrm{MeV} / \mathrm{fm}^{2}\right)$ & 72.1 & 98.8 \\
$a_{u u}^{c 0}(\mathrm{MeV})$ & -56.4 & -91.8 \\
$a_{u s}^{c 0}(\mathrm{MeV})$ & -63.0 & -116.8 \\
\hline \hline
\end{tabular}

quark model is nearly replaced by the vector-meson exchange in the extended chiral SU(3) quark model. In other words, the mechanisms of the quark-quark shortrange interactions in these two models are quite different.

Our previous work [4] has already shown that both these two sets of parameters can give a satisfactory description of the $S-, P-, D-, F$-wave $K N$ phase shifts. In this work, we use the same parameters to perform a preliminary study of the $\bar{K} N$ interaction by use of the RGM.

Fig. 1 shows the $S$-wave $\bar{K} N$ and $\pi \Sigma(I=0)$ scattering phase shifts calculated in the chiral $\mathrm{SU}(3)$ quark model. One sees that the phase shifts of both $\pi \Sigma$ and $\bar{K} N(I=0)$ are positive, which consequently means that their interactions are attractive, and besides, the effect of channel-coupling between $\bar{K} N$ and $\pi \Sigma$ is considerable, which is because of the big off-diagonal matrix elements of $\bar{K} N-\pi \Sigma$. We further solved the RGM equation for a bound state problem, and the results show that there is no $\pi \Sigma$ or $\bar{K} N$ bound state since the strengths of their attractions are not strong enough. To understand the contributions of various chiral fields to the $\bar{K} N$ interaction, in Fig. 2 we show the diagonal matrix elements of the generator coordinate method (GCM) calculation 10] in the chiral $\mathrm{SU}(3)$ quark model, which can describe the interaction between two clusters $\bar{K}$ and $N$ qualitatively. In Fig. 2. $s$ denotes the generator coordinate and $V(s)$ is the effective interacting potential between the two clusters. One sees that the attractive interaction of $\bar{K} N$ is dominantly provided by the $\sigma$ and $\sigma^{\prime}$ exchanges, but the strengths of the attractions are not very large, so that a bound state is unable to be formed. Nevertheless on the hadron level, the $\bar{K} N$ interaction has been widely investigated by use of the effective chiral Lagrange method [1]], and it is found that the $\bar{K} N$ interaction is attractive enough to form a bound state. In the chiral SU(3) quark model, if we take the parameters as the same as in the 


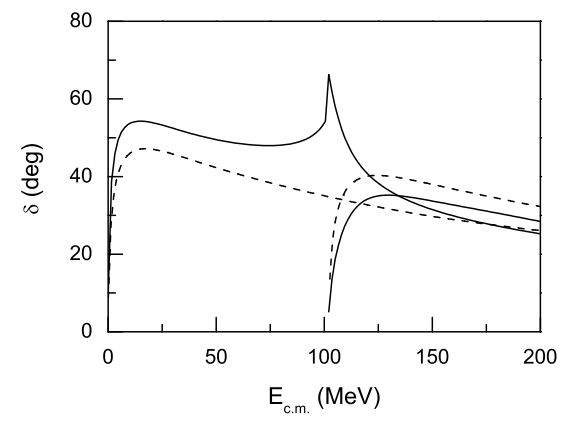

FIG. 1: $S$-wave $\bar{K} N$ and $\pi \Sigma(I=0)$ phase shifts in the chiral $\mathrm{SU}(3)$ quark model. The solid and dashed lines denote the results of single-channel and coupled-channels calculations, respectively. The $\bar{K} N$ phase shifts start at $E_{\text {c.m. }}=102 \mathrm{MeV}$.

$K N$ scattering study, we cannot get a $\bar{K} N$ bound state since the attraction strength is not strong enough. If we adjust the mass of $\sigma$ to a smaller value, the $\bar{K} N$ interaction will be much more attractive and it may be possible to get a $\bar{K} N$ bound state, but then the $K N$ scattering cannot be described simultaneously.

Fig. 3 shows the $S$-wave one-channel $\bar{K} N$ and $\pi \Sigma$ $(I=0)$ phase shifts in the extended chiral SU(3) quark model. The phase shifts indicate that both $\pi \Sigma$ and $\bar{K} N$ have very strong attractive interactions. Further study shows that such strong attractive interactions can make for $\pi \Sigma$ and $\bar{K} N$ bound states with the binding energies of about 4 and $12 \mathrm{MeV}$, respectively. Fig. 4 presents the diagonal GCM matrix elements of $\bar{K} N$, which denotes the contributions of various interaction terms. Comparing with Fig. 2. one sees that besides $\sigma^{\prime}$ and $\sigma, \rho$ and $\omega$ also offer strong attractions, which makes the $\bar{K} N$ interaction much more strongly attractive. The $\pi \Sigma$ is also much more attractive due to the contributions of vector meson exchanges. Such strong attractions consequently make for the $\pi \Sigma$ and $\bar{K} N$ bound states.

We know from Ref. [4] that for the $K N$ system, both the chiral $\mathrm{SU}(3)$ quark model and the extended chiral $\mathrm{SU}(3)$ quark model give similar contributions, though the

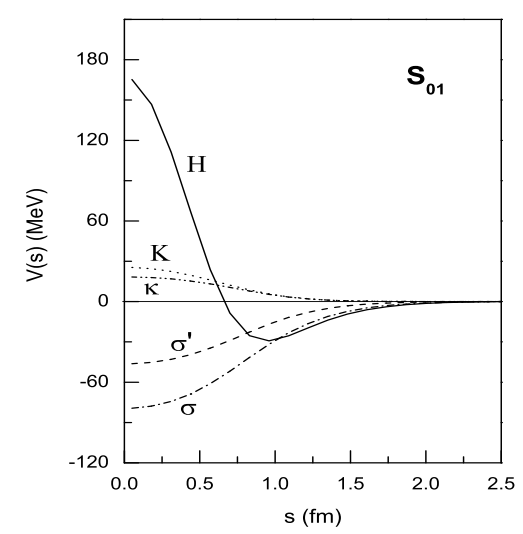

FIG. 2: GCM matrix elements for the $S$-wave $\bar{K} N$ system $(I=0)$ in the chiral SU(3) quark model.

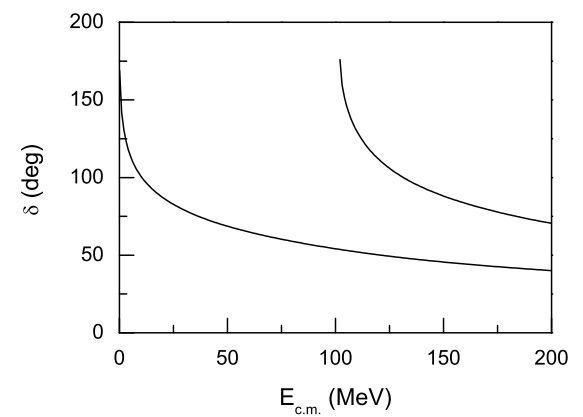

FIG. 3: $S$-wave $\bar{K} N$ and $\pi \Sigma(I=0)$ scattering phase shifts in one-channel calculation in the extended chiral SU(3) quark model. The phase shifts for $\bar{K} N$ scattering start at $E_{\text {c.m. }}=$ $102 \mathrm{MeV}$.

short-range interaction mechanisms are quite different in these two models. This means for the $K N$ system, the repulsion of OGE can be replaced by that of vector meson exchange. While for the $\bar{K} N$ system, OGE totally vanishes and vector meson exchange contributes strong attractions. Thus the results from those two models are quite different: neither $\pi \Sigma$ nor $\bar{K} N$ is bound in the chiral SU(3) quark model, while both $\pi \Sigma$ and $\bar{K} N$ are bound in the extended chiral SU(3) quark model. This means the attractions stemming from vector meson exchanges play a dominant role in the forming of the $\pi \Sigma$ and $\bar{K} N$ bound states, which is very similar to the works done at the hadron level with the effective chiral Lagrangian, where the lowest order chiral Lagrangian for meson-baryon interaction produces the Weinberg-Tomozawa term, which is nothing else than the exchange of vector mesons and does indeed produce attraction in the $\bar{K} N$ channel.

The appearance of the $\pi \Sigma$ and $\bar{K} N$ bound states in our model is very interesting, since on the hadron level all models using effective chiral Lagrangian have already reported two poles close to the $\Lambda(1405)$ : the higher one couples mostly to $\bar{K} N$ and the lower one mostly to $\pi \Sigma$ [11]. We further perform a $\bar{K} N-\pi \Sigma$ coupled-channels calculation, and the phase shifts are shown in Fig. 5. From this figure one sees that the $\bar{K} N$ bound state in the one-

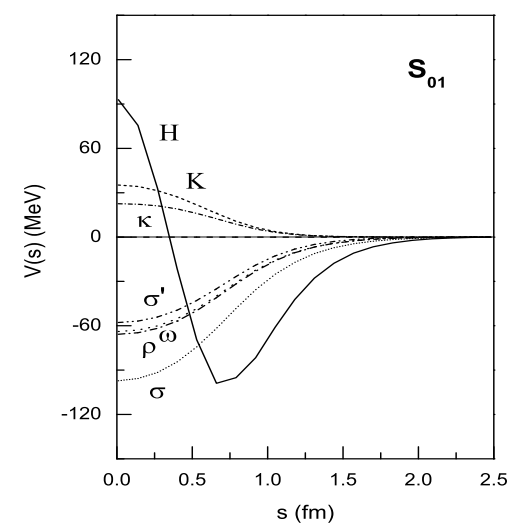

FIG. 4: GCM matrix elements for the $S$-wave $\bar{K} N$ system $(I=0)$ in the extended chiral $\mathrm{SU}(3)$ quark model. 


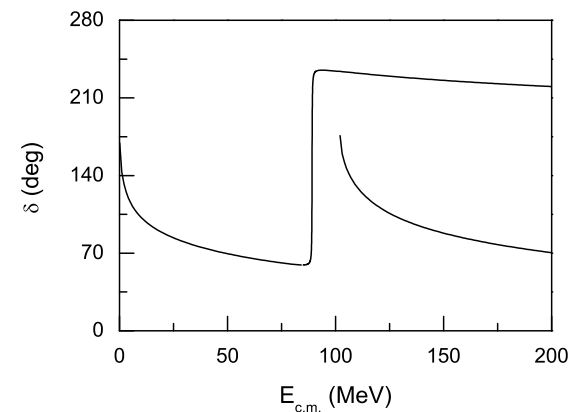

FIG. 5: $S$-wave $\bar{K} N$ and $\pi \Sigma(I=0)$ scattering phase shifts in coupled-channels calculation in the extended chiral $\mathrm{SU}(3)$ quark model. The $\bar{K} N$ phase shifts start at $E_{\text {c.m. }}=102 \mathrm{MeV}$.

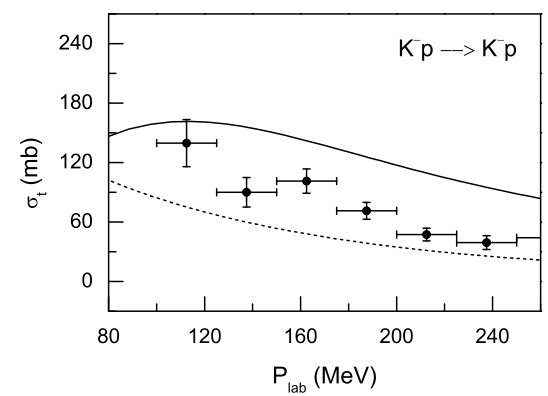

FIG. 6: Cross sections of $K^{-} p \rightarrow K^{-} p$. The dashed and solid lines represent the results from the chiral $\mathrm{SU}(3)$ quark model and the extended chiral SU(3) quark model, respectively. The experimental data are taken from Ref. [13].

channel calculation appears as a sharp $\pi \Sigma$ resonance just below the $\bar{K} N$ threshold in the coupled-channels investigation. In Ref. [12], Takeuchi and Shimizu claimed that the coupling of a $\pi \Sigma$ bound state and a three-quark state will make for a resonance near $\Lambda(1405)$. Thus it can be naturally expected that in the extended chiral $\mathrm{SU}(3)$ quark model another resonance with dominant $\pi \Sigma$ component would appear below the $\bar{K} N$ threshold with the inclusion of the coupling of a three-quark state to the $\pi \Sigma$ $\bar{K} N$ channels. If so, of great interest is that one comes to the same physics even using different approaches, i.e. the chiral quark model and the effective chiral Lagrangian method. Investigations along this line are planned for our next-step work.

Fig. 6 shows the $K^{-} p \rightarrow K^{-} p$ cross sections. One sees that the theoretical results are not far from the experimental data in both the chiral SU(3) quark model and the extended chiral SU(3) quark model. Nevertheless they are not good enough, which implies that the coupling of a genuine three-quark component to $\bar{K} N-\pi \Sigma$ should be included in order to improve the description.

In summary, we perform a preliminary study of the $\bar{K} N$ interaction using the same model, the same parameters and the same method as in the $K N$ phase shifts study [4]. It is found that the chiral SU(3) quark model cannot accommodate a $\bar{K} N$ bound state or a $\pi \Sigma-\bar{K} N$ resonance state, while in the extended chiral SU(3) quark model, both $\pi \Sigma$ and $\bar{K} N$ are bound and the latter appears as a $\pi \Sigma$ resonance in the coupled-channels calculation. We conclude that the vector meson exchanges are necessary to be included in the quark-quark interaction potentials if one tries to explain the $\Lambda(1405)$ as $\bar{K} N$ bound state or $\pi \Sigma-\bar{K} N$ resonance state. The $\bar{K} N$ cross sections and the effects of the three quark component will be studied in future work.

This work was supported in part by the National Natural Science Foundation of China (No. 10475087) and China Postdoctoral Science Foundation.
[1] Z.Y. Zhang et al., Nucl. Phys. A625, 59 (1997).

[2] L.R. Dai et al., Nucl. Phys. A727, 321 (2003).

[3] F. Huang, Z.Y. Zhang, and Y.W. Yu, Phys. Rev. C 70, 044004 (2004); F. Huang and Z.Y. Zhang, ibid. 70, 064004 (2004).

[4] F. Huang and Z.Y. Zhang, Phys. Rev. C 72, 024003 (2005).

[5] L.Y. Glozman and D.O. Riska, Phys. Rep. 268, 263 (1996); L.Y. Glozman, Nucl. Phys. A663, 103c (2000).

[6] N. Isgur, Phys. Rev. D 61, 118501 (2000); 62, 054026 (2000).

[7] F. Huang et al., Phys. Rev. C 71, 064001 (2005); F. Huang and Z.Y. Zhang, ibid. 72, 068201 (2006).

[8] L.R. Dai et al., Chin. Phys. Lett. 23, 3215 (2006).

[9] D. Zhang et al., Phys. Rev. C 75, 024001 (2007).

[10] K. Wildermuth and Y.C. Tang, A Unified Theory of the
Nucleus (Vieweg, Braunschweig, 1977).

[11] N. Kaiser, P.B. Siegel, and W. Weise, Nucl. Phys. A594, 325 (1995); E. Oset and A. Ramos, ibid. A635, 99 (1998); J.A. Oller, U.G. Meissner, Phys. Lett. B 500, 263 (2001); D. Jido et al., Nucl. Phys. A725, 181 (2003); T. Hyodo et al., Phys. Rev. C 68, 018201 (2003); C. GarciaRecio, M.F.M. Lutz, and J. Nieves, Phys. Lett. B 582, 49 (2004); B. Borasoy, R. Nissler, and W. Weise, Eur. Phys. J. A 25, 79 (2005); J.A. Oller, ibid. 28, 63 (2006); B. Borasoy, U.G. Meissner, and R. Nissler, Phys. Rev. C 74, 055201 (2006); V.K. Magas, E.Oset, and A. Ramos, Phys. Rev. Lett. 95, 052301 (2005).

[12] S. Takeuchi and K. Shimizu, arXiv: 0705.0565.

[13] W.E. Humphrey and R.R. Ross, Phys. Rev. 127, 1305 (1962). 